\title{
PDM Teaching Reform based on the Ontology Module and Data Management Platform
}

\author{
Yanchun XIA \\ Shanghai Second Polytechnic University, Shanghai, 201209, China \\ mollyxia@outlook.com
}

Keywords: PDM management platform, ontology modularization, practice teaching reform

\begin{abstract}
The course of the Product Data Management (PDM) is an Interdisciplinary course, which has the distinctive engineering and management characteristics. In order to satisfy the demand of teaching reform and researching of the PDM, the practice and applying is analyzed based on PDM software management platform. Based on the analysis, for the students' all-round development and improving the teaching effects and quality, the goal, main contents and the key problems of the course construction are proposed. In the teaching reform process, the ontology modularization method is introduced and organized in the way that there are several modules merged in it. This method not only eases the ontology development, but also helps to realize knowledge sharing, reusing and maintenance. In this platform, students can understand the real circumstance and apply it well.
\end{abstract}

\section{Introduction}

Production data management (PDM) course includes necessary elementary knowledge and contents for advanced technics in ME and IE fields. PDM technology is the core technology of production digital design and manufacturing based on data management. It can not only realize the design without blueprint, virtual machining and digital manufacturing, but also bring out the brand new design method and break through the traditional mode of the design concept. PDM is the 'steward' of the whole digital design process, which can transfer and share the information and data among the CAx modules.

With the demand of the up-to-date engineering and management talent, the specialty settings, talent cultivation and course construction etc. must be reformed and adjusted according to the careers fair[1]. In order to obtain the good teaching effects, the establishing of the software and hardware condition for practice teaching is very important. The share and analysis of the all kinds of teaching data, such as laboratory resources, course resources, classrooms, management files, students and teachers and so on, is very important and complex. How to collaborate effectively different domain data to have higher competitiveness capability, the computer and informatization technology is the key problem. In order to achieve common objectives, the resources, information and knowledge must be integrated in a platform. And for the data classification, the context ontology can be used, which is based on its capacity to enable a shared common understanding of a domain between different agents.

\section{Ontology Modules in the PDM teaching system}

\section{The principle and construction of module partition}

A domain ontology usually contains knowledge in many domains[2]. For example, in the field of the education, there involves many domains such as the teaching, the researching, the lab, the administration and so on. The relations in these subjects are very complex. How to partition a whole knowledge system into ontology modules is a key problem. Firstly, the principle of the module division should be easy to be understood. On the one hand the ontology system makes the computer understood the knowledge they processed, on the other hand, also the most basic, the ontology system should make the human understood. In this way, it is good for reusing, sharing, maintenance and extension of systems. Secondly, the division modules should have the characteristic that the 
knowledge is relative independent, which can reduce the coupling degree and communications between modules. That is in a whole ontology system $T$, set $\mathrm{R}^{\prime}$ and $\sigma^{\prime}$ should be as small as possible. The lower coupling degree between modules makes each module easy to reuse and share, just as it in the software engineering. Thirdly, the number of modules and relational modules should be as few as possible. Reducing the numbers of related modules can make the dependence between modules clearer and reduce the complexity of modules.

According to the principles of module division, in the education system of the university, the whole ontology system is divided into five modules about the PDM course teaching structure, the theory teaching, the researching, the lab and enterprise application system etc. It is easy to see that these modules themselves are all relative independent systems. In the whole ontology system, the relations of knowledge transfer between modules are quite clear. Fig.1 shows the system module and the knowledge transfer relations between modules. Such kind of domain module division result also obtained the approval of domain experts.

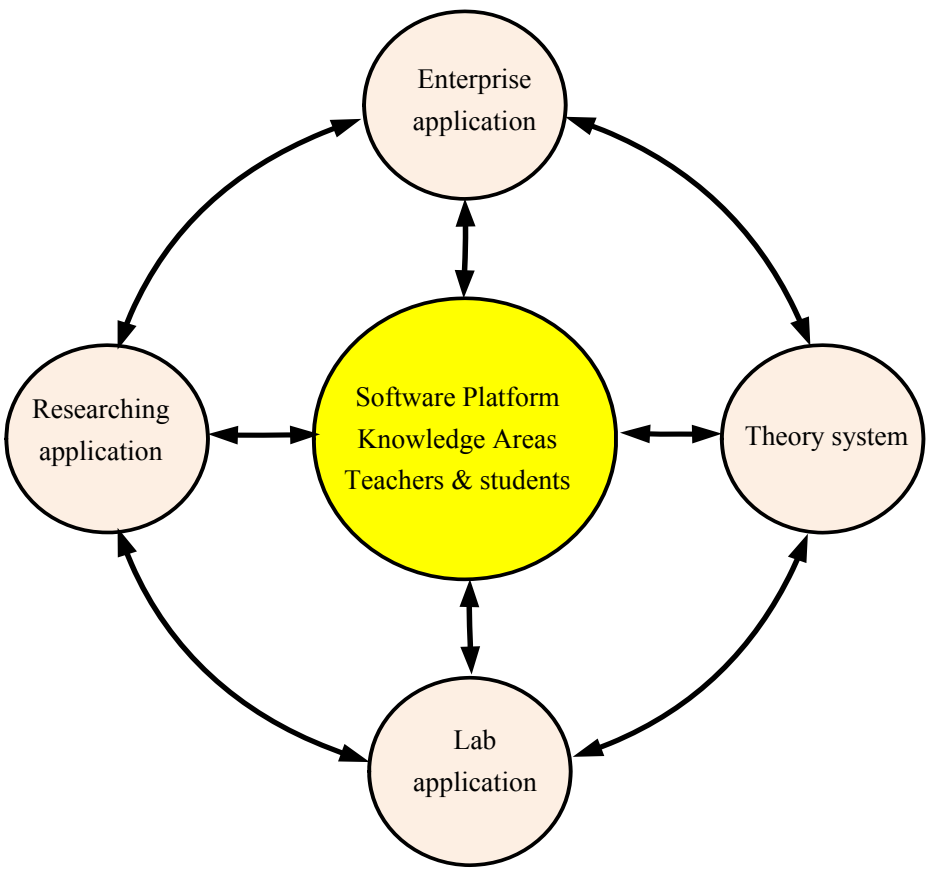

Fig.1 System module and knowledge transfer between modules

\section{Inference methods and the derivative mode of domain knowledge}

Nowadays, the most popular and easiest inference method of ontology is defining a group of logical axioms or rules in ontology knowledge base, by which the new facts can derive from the old facts[3]. The ordinary inference engine may draw the conclusions based on these rules or axioms, then bring the new knowledge and finally solve some simple problems. But these rules and axioms are specially suitable to a certain domain. Moreover the rules usually hide the connotative processing knowledge (such as rule order and union order), which may affect the practical applications of the inference engine at last. The connotative knowledge makes the rule base uncertainty. With the development of domains knowledge, it is hard to update.

Inference method base $\mathrm{Fi}$ of every ontology module $\mathrm{Ti}$ is established respectively using the inferential mode of separating the inference methods and the domain knowledge. Fi can be expressed as $\{\mathrm{fq}\}$. For query q, there are corresponding methods in Fi.

fq $(\mathrm{c} 1, \mathrm{c} 2, \ldots, \mathrm{cm}, \mathrm{q} 1, \mathrm{q} 2, \ldots, \mathrm{qn})$

where $\mathrm{c} 1, \mathrm{c} 2, \ldots, \mathrm{cm} \in \mathrm{Ci}$ expresses internal concepts in the Ti during the inference process. $\mathrm{q} 1$, $\mathrm{q} 2, \ldots \mathrm{qn}$ is the query to other modules knowledge. Pay attention, here we use q rather than $\mathrm{c}$, which expresses that qi doesn't quote the concepts of other modules directly, but carries on the query to other modules. In this way, the boundaries between modules are clear and one module is independent of the other. The method base needs to have the very good organization forms, with which we can carry on the index according to the different purposes and aims of methods. In a word, a good organization form is advantageous not only for the transfer of other methods but also for being flexible used by applications.

The method base solves some kinds of tasks using the domain knowledge. The method base provides the means of query and inference for obtaining the knowledge in the Ontology module. It also provides an external interface for the knowledge. So the application can get the knowledge conveniently without understanding the internal structure of the modules. The biggest advantage in using this kind of method base is reducing the coupling degree between ontology knowledge base and reasoning mechanism, strengthening the reusability and share. In addition, because the existing knowledge can be got through the special interface of domain knowledge bases, the independent 
method base can deal with data, complex logic reasoning issues and mathematical computation conveniently, needn't to consider the coupling problems of various conceptions. The method base abstracts and separates the processing knowledge from the domain knowledge, which makes the inference behavior and the task of domain knowledge in the system clearer and easy to update. As long as the knowledge in knowledge base doesn't involve the processing knowledge, the method base need not modify even the change of knowledge in the knowledge base. Similarly, the domain knowledge base need not change when the reasoning mechanism changes. What we need to do is change the method base.

\section{Educational resource management modeling}

\section{Educational resources polydimensional structure model}

The education system is a time-varying system, whose specific contents of each resource, planning modes are varied with the different period[4]. According to the educational characteristic and the thinking of the system engineering, the polydimensional structure model of the educational management platform is established. It shows as Fig.2.

The actual application system can be divided into three levels. One is the professional model level (the domain ontology in traditional significance), which is the set about certain domain knowledge. Another is the method level, which is the set of method that executes the reasoning process of the system to complete the task, and at the same time it is the query interface of each ontology module to other modules. And the last is the task level, which is the function set that the system must have. For the education reform system, the three levels structure can be showed in Fig.3.

The most important level is the method level, in which there is an applied task-oriented out of the system task management method base. All the method bases are imported directly or indirectly from this base. The task of task module can only transfer the methods in this base. The responsibilities of task method base are describing and separating a specific task, and transforming a whole task to the methods and their mutually cooperative relationships in some ontology module method bases. Then the task processing base transfers the methods of module method base to infer. When the inferences meet the knowledge which is based on other ontology module, the

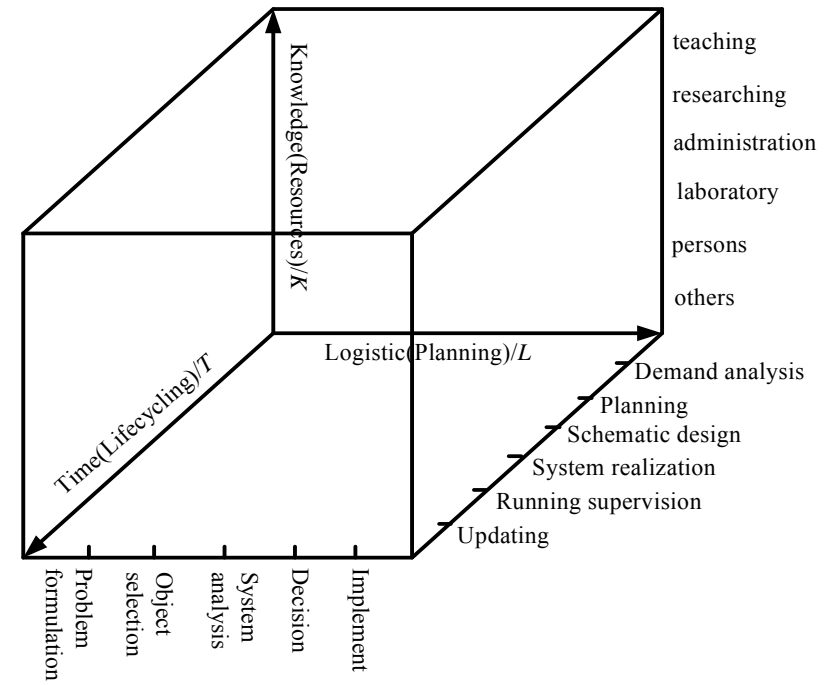

Fig.2 Three-dimension model of the teaching platform

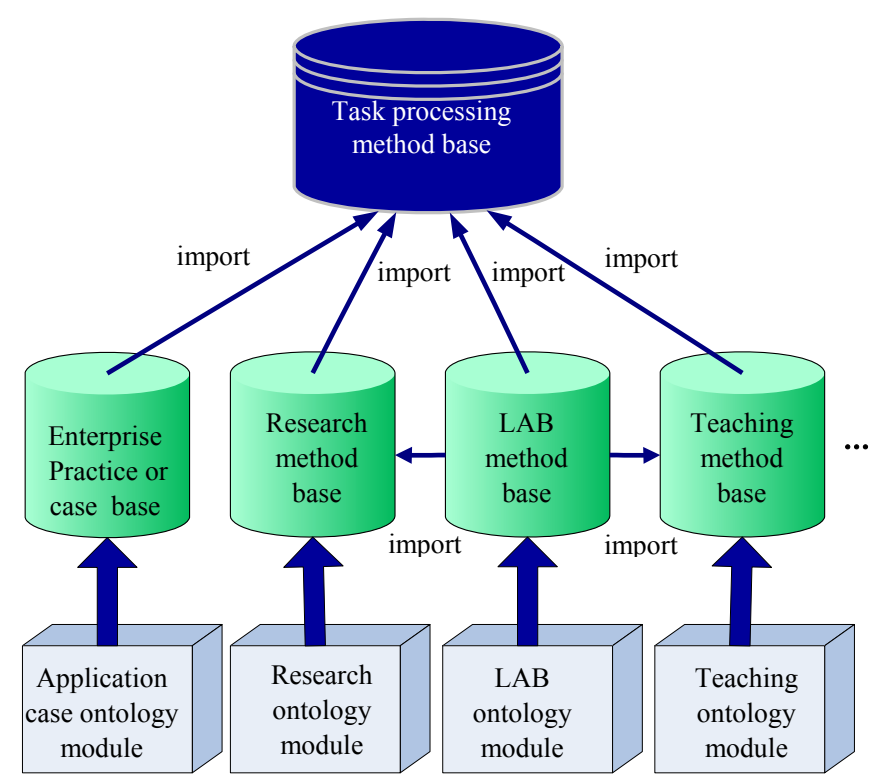

Fig.3 The structure of the system visible method of fi can be used to carry on the query. Each ontology module method base can be introduced into other module method bases. Each method base can only be introduced in a method base directly, but not circularly. All module method bases are introduced directly or indirectly into the duty processing method base. The relations of each 
module method base could import the other method base are similar to package, which avoided the traditional directly importing the methods in ontology knowledge base, reduced the coupling degree between the ontology modules greatly, and make the relations of them clearer. Each method base is equal to a knowledge adapter. When the module knowledge changed, as long as it didn't affect the query results, the method base didn't need to modify. In like manner, when the query requirements changed, the structure of knowledge base didn't need to adjust. The only demand is changing the corresponding methods in method base. When we need to reuse a certain module, it is very convenient for us to reuse it because the method we need is in the method base and the external interface of knowledge module is very clear. So, according to the above analysis, it is easy to maintain and reuse the modules for the system.

\section{Method description}

There are two methods in the method base, i.e. the atomic method and the composite method[5]. The atomic method can not be separated but can be transferred directly. The composite method is a method which constitutes by certain atoms and compound methods. Every method has one IPOE. The IPOE means Inputs, Outputs, Preconditions and Effects. Inputs and Outputs can be regarded as the transformation of data. Preconditions and Effects are the restrictions during the process, namely, the restriction conditions must be satisfied before the execution as well as the new restrictions

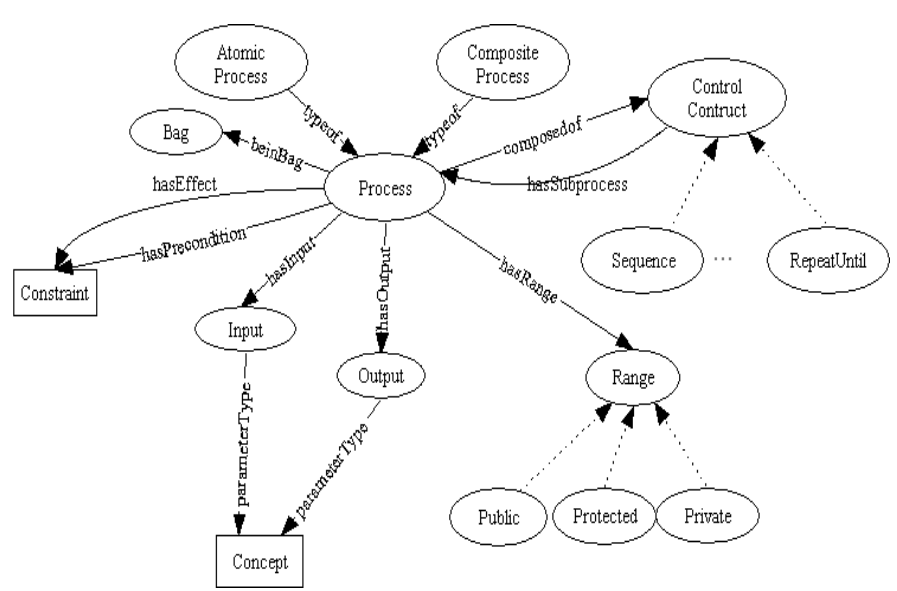

Fig.4 Method description execution produced. A composite method has a definition of ControlConstruct. ControlConstruct defines every executive order of every sub-process in the composite method. We use several flows of control in OWL-S here, such as Sequence, Split, Split+Join, Unordered, Choice, If-Then-Else, Iterate, Repeat-Until etc.

Fig. 4 is the structural drawing of each attribute of the method. Bag was the URI of the method base. The relations of beinBag expressed the method base that certain method was in. Range is the attribute of action scope of the method. In this way, the methods in the method base may be described according to the form of ontology conception. The method itself is regarded as a resource. The characteristics of the method, such as the inputs and outputs of method, the preconditions and the effects and so on, can be expressed by various relation forms. The structural drawing expresses that it makes application and other methods understand the services which provided by methods using ontology. However, it is not merely the expression contains of rectangles in the figure 3 . The rectangles just express the resources in the module of ontology knowledge base.

It can be seen that there is still certain coupling degree between the modules at present, which mainly manifested at the transferring between different methods. For example the methods signed as public or protected can be used by other method bases, especially the public method. The public method not only causes the system more complex, but also makes great affection on the efficiency of inference in the distributed or large-scale ontology. In view of this point, the system can save the knowledge of other modules to the ontology module base, which makes the inference in the module come true. But this method is very sensitive to the revision of the ontology module. One side, after the query is completely edited, the correctness of inference is ensured under the situation that the queried module structure is not changed. On the other hand, the edit results of the interface are not affected by every change of the module structure. It appeared only when the conception which used in the query process or the class structure containing queries changed. In the former instance, we possibly need to consider redefining the queries, but for the latter, we must reedit the interface. In order to make sure whether the editing axioms keep on efficiency, we may adopt a mechanism of revision and detection which based on a kind of revision classification to the ontology. This kind of factors contained the affections of revision to the framework of class as well as the position of the class in the class structure. We can still adopt a clear expression mode which shows the dependency relationship between modules in order to make the corresponding change very easily according to the change of related part of certain module. 


\section{The realization of the management platform}

The structure of the object-oriented for unified educational resource management platform system is shown in Fig.5. The platform includes the resource database level, resource model level, data mapping and sharing level, server lever and application level. The resource database can be used and shared by PDM system, ERP system and some other software systems. The course resource information, research project information, the Electronic Documents of the graduation projects, administration files etc. can all be included in the database. In the application level, the functions of the resource modeling, inquiry, statistical summary, Rights Management, etc. can be realized. Based on the

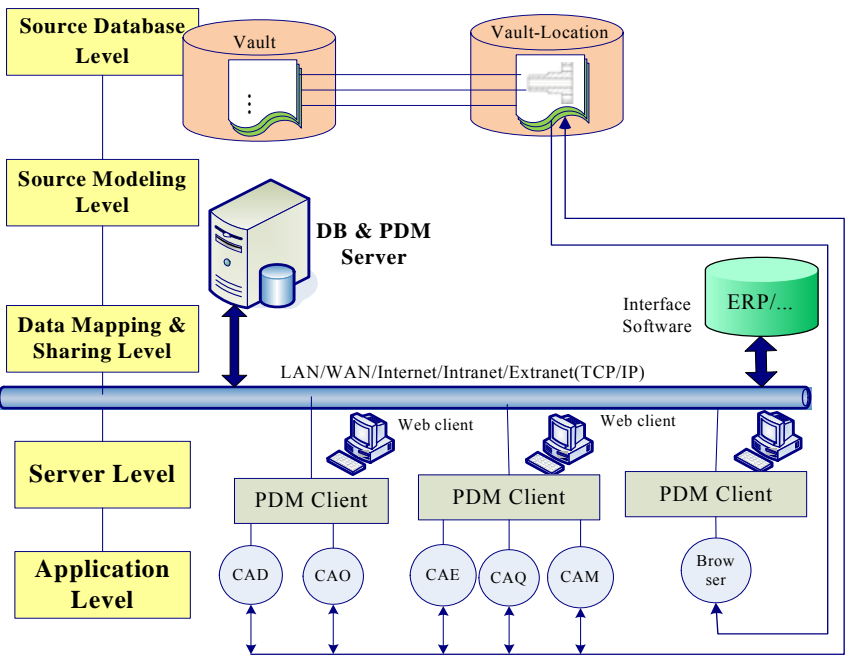

Fig.5 The frame of the faculty management platform construction of the ontology modularization model of the educational management platform, the frame of the faculty management platform was established, and then the customized education data management system was designed and implemented.

\section{Summary}

Based on the ontology modularization unified educational resource management platform is the effective approach to realize the efficient planning and management as a whole. First, the modularization makes the division of work clear during the development. During the development process of the ontology especial the large-scale ontology, it needs the cooperation of expert groups in different fields. After the modularization, the work obtained the standard, the responsibility is clearly defined, and the construction achievement is easy to integrate. Second, the modularization reduces the complexity of the system. The relation of conceptions existing in each module can simplify the design and maintenance of modules. The method base can achieve the communication from one module to another. Because the coupling degree in the module is lower, it is easy to change the knowledge in the module without changing the external interface. In this way, the updating maintenance becomes extremely convenient. Third, the modularization is easy to be shared and reused in the system. However, each module in our system is a quite integrated theory system, in which each module can be reused solely. Because each method base of ontology module and knowledge base are independent, we can redesign the method base to adapt the new application demands without changing the knowledge base. It provides favourable guarantee for the sharing and reusing of modules. At last, although the ontology is in order to achieve the knowledge sharing, for the consideration of privacy, security or the convenience of management in practice, we might hidden some parts of ontology selectively. Using the modularizing technology, the scope attribute in method base can solve this problem well.

Compared to the modularization of software engineering, the modularization of ontology method base isn't quite mature, and still has lots of problems to be solved. At present, the management platform is implemented in the developing and perfection stage, the ontology modularization modelling method and the subsequent secondary development functions developing can be further researched and practised.

The PDM course construction is implemented by teaching goals, content system, method system and management system. In the teaching practice process, the subsystems not only play out their different roles and functions, but also coordinate mutually to realize the global aims. The course 
construction analysis and application is combined with the talent market closely. The construction experiences can be popularized further.

\section{Acknowledgements}

The research work was supported by Shanghai education science research projects under Grant No. B51JD12R002 and teaching reform project under Grant No. A01GY13F027.

\section{References}

[1] Xia Yanchun Ye Weidong et al. Teaching Planning and Design of the 'Production and Operation Management'. 2010 International Conference on Education and sports Education. Wuhan, China. Vol.1, 149-152, 2010

[2] Yan Ye, Dong Yang, et al. Ontology-based semantic models for supply chain management. The International Journal of Advanced Manufacturing Technology, Vol.37, No. 11-12, 1250-1260, 2008

[3] Shijun Yu, Stefano Spaccapietra. A knowledge infrastructure for intelligent query answering in location-based services. GeoInformatica, Vol. 14, No. 3, 379-404, 2010

[4] Yue Zhang and Yongjiang Zhu. The education connotation of the engineering applied undergraduate and its cultivation mode construction. Journal of national academy of education administration (Chinese), 7-9,39, 2009

[5] David Bell, Sergio de Cesare, et al. A framework for deriving semantic web services. Information Systems Frontiers, Vol.9, No. 1,69-84, 2007 